Anales de Geografía de la Universidad Complutense ISSN: 0211-9803

\title{
Propuesta sostenible para mitigar los efectos climáticos adversos en una ciudad costera de Argentina
}

\author{
Federico Ferrelli ${ }^{1}$; María Cintia Piccolo ${ }^{2}$ \\ Recibido: 3 de marzo del 2016 / Enviado a evaluar: 22 de mayo del 2016 / Aceptado: 18 de julio del 2016
}

Resumen. Los indicadores de sostenibilidad climática constituyen herramientas fundamentales para complementar las políticas de ordenamiento del territorio urbano y pueden beneficiar la calidad de vida sus habitantes. En el presente trabajo se diseñó un indicador climático urbano para la ciudad de Bahía Blanca considerando variables meteorológicas y análisis de la percepción social. El mismo permitió delimitar la ciudad en cuatro regiones bien diferenciadas entre sí. A partir de entonces, se realizó una propuesta sostenible para mitigar los efectos adversos del clima a partir de la aplicación del método DPSIR. Las mismas estuvieron destinadas a mejorar las condiciones de vida de la población. Los resultados permitieron considerar que una pronta implementación de la misma junto con una activa participación de los actores sociales y los tomadores de decisiones es necesaria para mejorar las condiciones actuales en la que se encuentra la ciudad. Con las medidas propuestas, la población local sabrá cómo actuar ante la ocurrencia de distintos eventos extremos, eventos de desconfort climático, etc. Al ser un método sencillo, la metodología aplicada en este estudio puede replicarse en otras ciudades del mundo con el objetivo de mejorar la calidad de vida de los habitantes.

Palabras clave: Indicador climático urbano; sostenibilidad; actores locales; Bahía Blanca.

\section{[en] Sustainable proposal to mitigate the adverse climatic effects in a coastal city of Argentina}

\begin{abstract}
Sustainable climatic indicators are useful tools to complement urban land use planning and could be beneficial for the quality of life. In this study, it was designed an urban-climatic-indicator for Bahía Blanca city considering meteorological variables and perception analyses. It allows delimiting four urban regions. Then, a sustainable proposal was made in order to mitigate the adverse climatic effect from the application of DPSIR method. Results permitted considering that an early implementation of this proposal and the active participation of stakeholders and decision makers is needed to improve the current conditions of the city. With the measures proposed inhabitants know how to respond in case of

1 Instituto Argentino de Oceanografía (IADO-CONICET). Departamento de Geografía y Turismo. Universidad Nacional del Sur.

E-mail: fferrelli@criba.edu.ar

2 Instituto Argentino de Oceanografía (IADO-CONICET). Departamento de Geografía y Turismo. Universidad Nacional del Sur.
\end{abstract}


the occurrence of various extreme meteorological events, discomfort conditions, etc. As a simple method, the methodology used in this study can be replicated in other cities worldwide with the aim of improving the quality of life of the inhabitants.

Key words: Urban-climate-indicator; sustainability; stakeholders; Bahía Blanca.

\section{[fr] Proposition durable pour limiter les effets climatiques indésirable dans une ville cotiere de l'Argentine}

Résumé. Les indicateurs de durabilité climatiques constituent des outils fondamentaux pour améliorer les politiques d'aménagement des territoires urbains et pourraient être bénéfiques pour l'amélioration de la qualité de vie des habitants. Dans cette étude a été conçu un indicateur climatique urbain pour la ville de Bahía Blanca en considérant des variables météorologiques ainsi que l'analyse de sa perception sociale. Il permet de délimiter la ville en quatre zones, différentes entre elles. Par la suite, une proposition durable a été présentée afin d'atténuer les effets climatiques défavorables, grâce à l'application de la méthode DPSIR. Ces dernières étaient destinées à améliorer les conditions de vie de la population. Les résultats ont permis de considérer qu'une mise en œuvre rapide de cette proposition, alliée à la participation active des personnes et des décideurs, est nécessaire pour améliorer les conditions actuelles de la ville. Avec les mesures proposées, les habitants sauront comment réagir en cas de divers événements météorologiques extrêmes, des conditions d'inconfort climatique, etc. En tant que méthode simple, la méthodologie utilisée dans cette étude peut être reproduite dans d'autres villes de par le monde, dans le but d'améliorer la qualité de la vie des habitants.

Mots clés: Indicateur climatique urbain; la durabilité; les parties prenantes; Bahía Blanca.

Cómo citar. Ferrelli, F. y Piccolo, M.C. (2016): Propuesta sostenible para mitigar los efectos climáticos adversos en una ciudad costera de Argentina. Anales de Geografia de la Universidad Complutense, 36(2), 281-306.

Sumario. 1. Introducción. 2. Metodología. 3. Resultados y discusión. 3.1. Factores determinantes (drivers). 3.2. Presiones (pressueres). 3.3. Estado ambiental (state). 3.4. Impactos (impacts). 3.5. Estado climático de la ciudad de bahía blanca. 3.6. Propuesta sostenible para la ciudad (responses). 3.6.1. Referidas a la conservación de los espacios verdes. 3.6.2. Referidas a la reglamentación de las construcciones. 3.6.3. Referidas a la capacitación social. 4. Conclusiones. 5. Referencias bibliográficas.

\section{Introducción}

Las ciudades se encuentran en constante crecimiento generando impactos sobre el microclima urbano, afectando a la salud de la población, los ecosistemas urbanos, los recursos hídricos y la disponibilidad de agua (Vilani y Sanchez, 2013). Por ello, el proceso de urbanización planificado ha surgido como un mecanismo para afrontar esta situación. Este se basa no sólo en variables sociales y económicas sino que también incluye las ambientales, teniendo en cuenta el clima de la ciudad ya que es un elemento fundamental para lograr la sostenibilidad en los modelos desarrollados. En este contexto el clima urbano es uno de los recursos limitantes para el desarrollo de la ciudad ya que puede exponer a los ciudadanos a situaciones de riesgo ya sea por exceso de calor, frío, inundaciones etc. (Cuadrat et al., 2014).

El Panel Intergubernamental del Cambio Climático (IPCC) señala que a escala global la temperatura del aire se incrementará. Esta situación será más intensa en las 
ciudades por la generación de las Islas de Calor. Por este motivo se sugiere poner el estudio del clima urbano en el centro de atención para prevenir los cambios y definir estrategias de adaptación. En el contexto de cambio climático actual, las ciudades son los principales ejes de estudio debido a que en las mismas se concentran las mayores emisiones de gases de efecto invernadero y además son las fuentes artificiales de calor. La gestión del territorio debe estar orientada al desarrollo de infraestructura adecuada, a la provisión de agua potable, a la confortabilidad de los ciudadanos y al beneficio de su salud (Cuadrat et al., 2014). Las arquitecturas bioclimáticas, como las terrazas verdes, se originaron para mejorar el confort climático de las personas. Las mismas son formas de adaptación del ser humano al clima (Ballesteros Zapata, 2013).

Las ciudades requieren de información que ayude a orientar un planeamiento climático sostenible (Franco Silva y Pérez Salazar, 2008). En la Agenda 21 se propuso implementar indicadores de desarrollo sostenible para generar una base sólida para la toma de decisiones (CSD, 2007; Zulaica y Tomadoni, 2015). Actualmente las urbanizaciones costeras en crecimiento generan una degradación del ambiente contribuyendo a intensificar el cambio climático actual. En la literatura se encuentran trabajos realizados en las ciudades costeras a partir del método DPSIR (por sus siglas en inglés, Driver-Pressure-State-Impact-Response framework) (Agencia Europea de Medio Ambiente, 1999 en Jago-On et al., 2009). Este es una herramienta que considera distintos procesos ambientales con la finalidad de desarrollar propuestas de gestión apropiadas a cada espacio. Su desarrollo engloba variables de tipo social, económico y ambiental (Gabrielsen y Bosch, 2003; Kristensen, 2004; Sekovsky et al., 2012).

En este trabajo, el área de estudio fue la ciudad de Bahía Blanca localizada al sudoeste de la provincia de Buenos Aires, Argentina (Figura 1). Es una urbanización costera que durante los últimos 30 años ha experimentado una notable expansión urbana (11.000 ha) y crecimiento poblacional (67.000 habitantes, teniendo más de 300 mil habitantes en 2016). Los ciudadanos han percibido cambios en el clima y en la periodicidad de los eventos extremos y han expresado los problemas ambientales que más los afectan. Además, el decrecimiento de la biomasa urbana impactó aumentando la temperatura del aire $\left(0,9^{\circ} \mathrm{C}\right)$ y reduciendo la humedad relativa $(6 \%)$ (Ferrelli et al., 2016a). Por lo mencionado, el objetivo de este trabajo fue diseñar un indicador urbano para delimitar regiones con un comportamiento climático similar en la ciudad de Bahía Blanca que contemple además la percepción de la población. La aplicación del mismo permitió enmarcar una propuesta sostenible para mitigar los efectos adversos del clima y con ello orientar medidas destinadas a mejorar la calidad de vida de la población. 
Figura 1. Localización de la ciudad de Bahía Blanca y las estaciones meteorológicas.
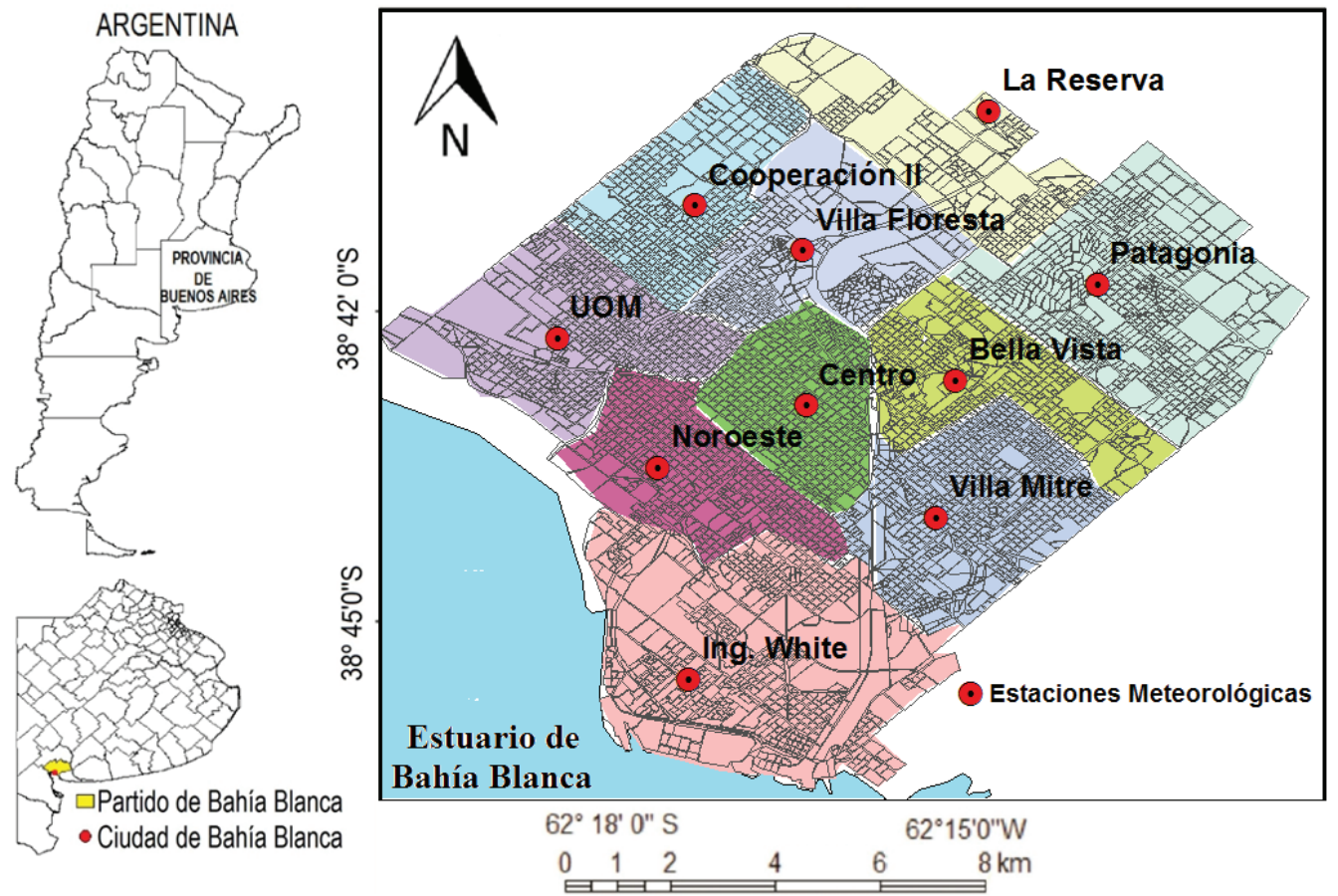

Fuente: Elaboración propia, 2016.

\section{Metodología}

El método DPSIR se aplicó con el fin de diseñar una propuesta para mitigar los efectos adversos climáticos. El mismo se basa en la recopilación de información de la sociedad y del ambiente con el objetivo de interpretar el efecto de las fuerzas externas sobre los posibles cambios sociales y económicos. Este método permitió describir las inter-relaciones entre la sociedad y su ambiente. Para su aplicación se consideraron distintas variables y se contextualizaron teóricamente los factores que componen el método (Figura 2).

Para la descripción del ambiente urbano de la ciudad de Bahía Blanca se recopilaron variables cualitativas y cuantitativas. Las mismas sirvieron de base para orientar y diseñar la propuesta. Las variables cualitativas se analizaron a partir de datos obtenidos con cuestionarios (Total: 177). Los mismos estuvieron dirigidos a grupos de vecinos próximos a los barrios considerados en esta investigación por lo que los resultados de cada uno de ellos reflejan la percepción de grupos de personas. La forma de aplicación se hizo mediante un muestreo de tipo no probabilístico por 
cuotas. En el mismo se consideraron en las muestras grupos de personas previamente seleccionadas. Los mismos estuvieron orientados a toda la población de la ciudad y generaron información sobre la percepción del clima, intensidad de distintos eventos extremos meteorológicos, necesidad de espacios verdes, aumento o disminución del tránsito vehicular y las edificaciones, entre otras. Las respuestas a las preguntas fueron mayoritariamente cerradas con opciones Muy Bueno, Bueno, Regular, Malo y Muy Malo teniendo en cuenta la metodología aplicada en otros estudios de percepción urbana como el de Ferrelli et al. (2016a). Estas categorías se utilizaron para ponderar las variables cualitativas.

Figura 2. Esquema DPSIR para la ciudad de Bahía Blanca.

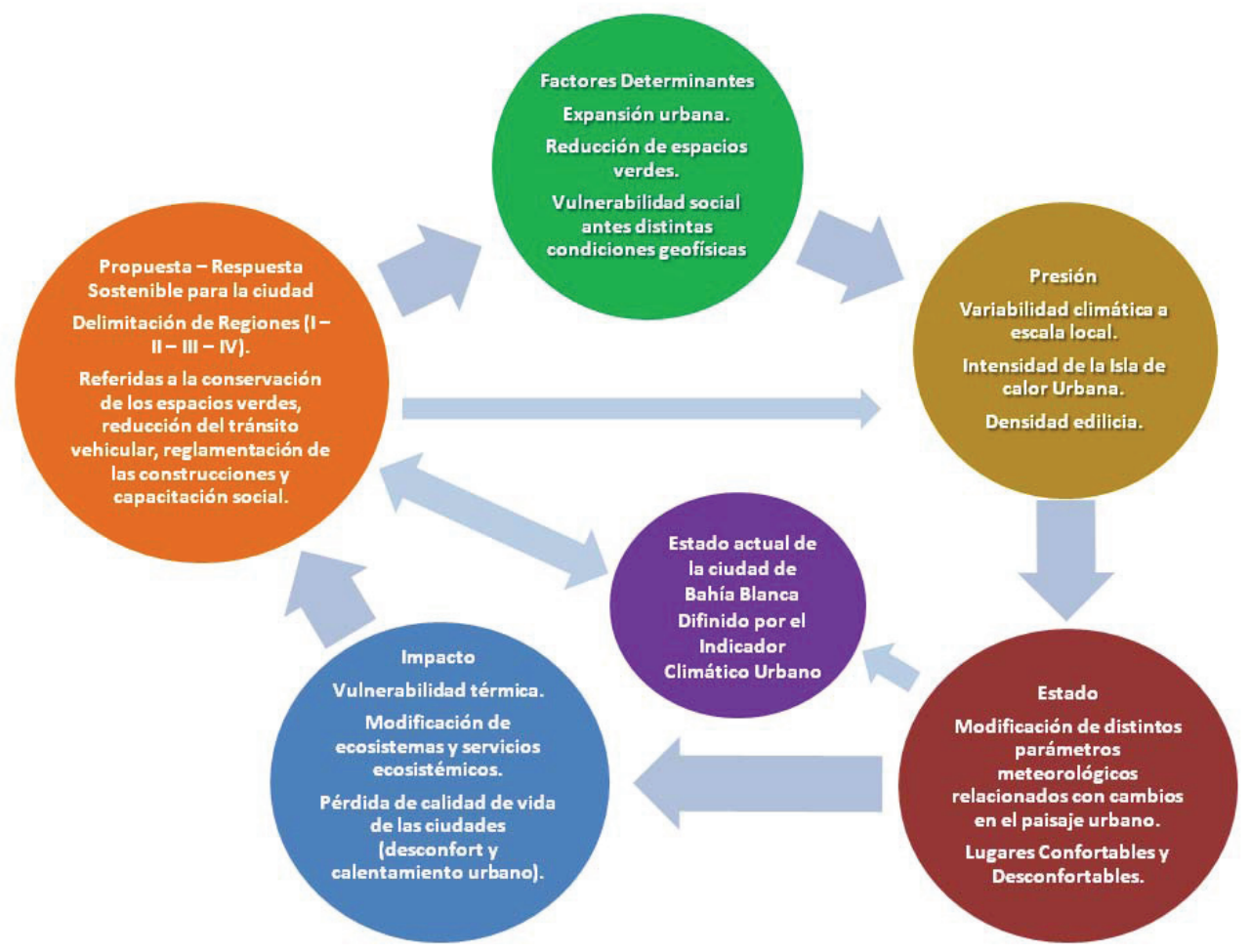

Fuente: Modificado de Sekovski et al., 2012.

Las cuantitativas se obtuvieron mediantes distintos instrumentos de medición. Se calculó la Intensidad de la Isla de Calor Urbana de la ciudad de Bahía Blanca y se analizó su evolución durante los últimos 30 años (Ferrelli et al., 2016b). Además, se diseñaron instrumentos de medición en alta frecuencia para registrar datos meteorológicos de temperatura del aire y humedad relativa en distintos sectores de la 
ciudad. Los mismos se instalaron en 10 barrios equidistantes entre sí (Figura 1) durante 408 días (octubre 2013-diciembre2014). La distancia entre cada uno de ellos consideró que se cubriera la mayor cantidad de superficie urbana posible. Las mismas se colocaron en sitios con características similares: a la sombra, protegidas de la lluvia, con circulación de aire y a 2 metros de altura. El intervalo de muestreo fue de 30 minutos.

Una vez que las estaciones meteorológicas fueron instaladas, se las renombró para su análisis considerando el espacio que representaron. Esta nomenclatura tuvo en cuenta el nombre del barrio de la ciudad en el que fueron instaladas. De esta forma se denominaron:

1) Centro (C): Se localizó en el centro de la ciudad. Representó un espacio urbano de alta densidad de edificios en altura, con gran cantidad de tránsito vehicular, comercios, densidad de población y escasez de espacios verdes.

2) Villa Mitre (VM): Se localizó en el centro-sudeste de la ciudad. Representó un espacio construido con alta densidad de viviendas.

3) Bella Vista (BV): Representó las condiciones de un espacio verde. Se instaló sobre el Parque Independencia localizado en el este de la ciudad.

4) Villa Floresta (VF): Se localizó en las proximidades del Parque de Mayo. Representó las condiciones de los espacios urbanizados próximos al mismo.

5) Patagonia (Pat.): Fue instalada en un barrio-parque. Se localizó en el noreste de la ciudad. Representó un espacio urbano con gran densidad de arbolado urbano, presencia de calles pavimentadas y sin pavimentar y con una densidad de edificación menor a las anteriormente descritas.

6) Cooperación II (C II): Se localizó en el oeste de la ciudad. Representó un barrio residencial con poco tránsito vehicular, menor densidad de construcciones y calles pavimentadas.

7) Noroeste (NW): Se localizó en el sudoeste de la ciudad y representó un espacio con construcciones residenciales de escasa altura.

8) UOM: Se localizó en el oeste-sudoeste de la ciudad, representó un barrio residencial. Fue un espacio similar al de Cooperación II pero con menor densidad de edificaciones, calles sin asfaltar y mayor número de terrenos al descubierto.

9) Ingeniero White (IW): Fue instalada sobre la zona portuaria de la ciudad. Se caracterizó por su cercanía al mar, escasa densidad de construcciones y abundante presencia de terrenos baldíos o al descubierto.

10) La Reserva (LR): Se denominó de esta manera a la estación perteneciente al CERZOS. Representó un espacio peri-urbano y periférico dado que durante el período de estudio no presentó edificaciones que pudiesen modificar los parámetros de humedad relativa y temperatura del aire.

A la información recopilada por estas estaciones se le aplicaron índices de confort estival e invernal y se identificaron distintos eventos extremos ocurridos durante el período de medición (Ferrelli et al., 2015a; Ferrelli, 2016). Para la ponderación de estas variables se consideraron los aportes de Berenson y Levine (1996). Se llevaron 
los valores a una distribución normal $\mathrm{N}(0,1)$ a partir de la aplicación de distintas pruebas para verificar la existencia de la normalidad. Para probarlas se aplicaron las pruebas de Kolmogorov Smirnov y Shapiro Wilks con un $\alpha 0,05$. Además, se realizaron los gráficos de QQ-Plot e histogramas de frecuencia y se calcularon la asimetría y el coeficiente de Curtosis. En la tabla 1 se presentan los valores medios para cada variable considerada en cada barrio.

Toda la información recopilada fue tabulada en un SIG considerando los 10 barrios (Figura 1). Se realizaron análisis espaciales con los datos obtenidos. Además se consultaron mapas de coberturas del suelo, distribución espacial de la temperatura calculada con imágenes satelitales, del índice de vegetación NDVI, de la ICU y de los efectos de distintos eventos meteorológicos extremos considerando los estudios previos que se mencionan a continuación.

Con toda esta información se diseñó un Indicador Climático Actual de Bahía Blanca (IClimU). El mismo se constituyó con las siguientes variables:

1. Densidad edilicia. Los edificios modifican la circulación del viento acentuando la presencia de aire contaminado por las actividades urbanas. Por este motivo se consideró con un valor 1 a aquellos sectores de la ciudad de Bahía Blanca en donde la densidad edilicia fue mayor y 0 a las que tuvieron menor (Ferrelli et al., 2015b, Tabla 1).

2. Espacios Verdes. Se estableció que los mismos son elementos importantes para el ambiente urbano ya que poseen la capacidad de reducir la carga radiante, favorecen la infiltración de las precipitaciones y permiten la realización de actividades recreativas. Este parámetro incluyó la importancia relativa que los encuestados le adjudicaron a los espacios verdes de los distintos sectores urbanos. Las zonas con mayor densidad de vegetación se las representó con una valor 0 y la escasez de los espacios verdes generó valores cercanos a 1 (Ferrelli et al., 2015b, Tabla 1).

3. Isla de Calor Urbano. La intensidad de la ICU fue distinta según la época del año (verano o invierno) y según la localización. Se adquirieron datos en 1985 y 2014. Estos valores se normalizaron y se llevaron a valores entre 0 y 1 (Piccolo y Capelli de Steffens, 1985, 1987; Ferrelli et al., 2016b, Ferrelli 2016, Tabla 1).

4. Temperatura de Superficie Terrestre (TST). Fue analizada desde el concepto de la expansión urbana y la rugosidad del paisaje urbano. Permitió inferir el crecimiento edilicio a partir de la rugosidad del espacio y la generación de sombras que originan valores bajos del parámetro. Para establecer su cuantificación se tuvo en cuenta el comportamiento que la TST. Donde este parámetro presentó mayor variación espacial los valores fueron cercanos a 1 y cuando la variación fue mínima, cercanos a 0 (Ferrelli et al., 2015b, Tabla 1).

5. Temperatura del aire. Los sectores urbanos altamente edificados presentaron valores de temperatura mayores que el resto de la ciudad. Aquellos barrios en donde los registros fueron mayores se las identificó con el valor 1 (Ferrelli, 2016, Tabla 1). 
6. Humedad Relativa. Se consideró que los espacios con mayor humedad relativa podrían verse favorecidos por la presencia de árboles y cercanía al mar por ello se los ponderó con 1 (Ferrelli, 2016, Tabla 1).

7. Efecto de los eventos extremos. Se consideraron olas de calor, olas de frío, vientos intensos del norte y del sur, heladas y nieblas. Los sectores urbanos más afectados se los identificó con 1 y los menos con 0 (Ferrelli, 2016, Tabla 1).

8. Confort climático estival. Se cuantificaron los días con desconfort en cada sector urbano. Aquellos con mayor número de días de desconfort fueron las que presentaron el valor 1. Este criterio incluyó los índices Humidex (Weather Service of Environment Canada, 2001) y Termo-higrométrico (THI) (Thom, 1959) y Termo-anemométrico (P) (Siple y Passel, 1945; Capelli de Steffens et al., 2005, Ferrelli, 2016, Tabla 1).

9. Confort climático invernal. Se estandarizaron los datos de confort obtenidos con el índice de Temperatura Equivalente (Te) de Quayle y Steadman (1999) (Ferrelli, 2016, Tabla 1).

10.Percepción sobre la calidad ambiental. Se consideró 0 al estado ambiental Muy Bueno, 0,25 a Bueno, 0,5 a Regular, 0,75 a Malo y 1 a Muy Malo (Ferrelli et al., 2016a, Tabla 1).

11.Percepción sobre el Incremento del tránsito Vehicular. El criterio adquirido fue 0 para el crecimiento Sin cambios, 0,5 para el Medio y 1 para Alto (Ferrelli et al., 2016a, Tabla 1).

12.Aumento de las edificaciones. Se consideró 0 al aumento Muy Bajo, 0,25 al Bajo, 0,5 al Medio, 0,75 al Alto y 1 al Muy Alto (Ferrelli et al., 2016a, Tabla 1).

13.Problemas ambientales. Los encuestados identificaron distintos problemas ambientales en los diferentes sectores de la ciudad. Aquellas que presentaron mayor número se identificaron con 1 y el 0 correspondió a las que no lo tuvieron (Ferrelli et al., 2016a, Tabla 1).

14.Sensación térmica urbana. Incluye la sensación de la población ante los cambios térmicos analizados. El valor 1 fue para Gran aumento térmico, 0,66 El aumento fue moderado, 0,33 Aumento leve, 0 La temperatura disminuyó (Ferrelli et al., 2016a, Tabla 1).

15.Eventos extremos percibidos. La cantidad de eventos extremos percibida por los habitantes de cada barrio fue distinta. Aquellos que presentaron mayor número fueron los que adquirieron 1 y los que no presentaron ninguno, 0 (Ferrelli et al., 2016a, Tabla 1). 
Tabla 1. Valores medios estandarizados para la elaboración del Indicador Climático Actual para el período octubre 2013-diciembre2014 para cada barrio considerado ${ }^{3}$.

\begin{tabular}{|c|c|c|c|c|c|c|c|c|c|c|}
\hline Variables & LR & C & VF & UOM & IW & $\mathbf{V M}$ & BV & Pat & NW & C II \\
\hline Edificación & 0 & 1 & 0.8 & 0.2 & 0.2 & 0.9 & 0.5 & 0.4 & 0.5 & 0.2 \\
\hline Espacios Verdes & 0 & 0.8 & 0.1 & 0.5 & 0.5 & 0.7 & 0.1 & 0.3 & 0.7 & 0.5 \\
\hline TST & 0.4 & 0.9 & 0.8 & 0.5 & 0.6 & 0.9 & 0.4 & 0.4 & 0.6 & 0.5 \\
\hline Temperatura & 0.2 & 0.8 & 0.6 & 0.86 & 0.4 & 0.4 & 0.3 & 0.4 & 0.42 & 0.4 \\
\hline Humedad relativa & 0.5 & 0.5 & 0.4 & 0.86 & 0.2 & 0.73 & 0.3 & 0.2 & 0.46 & 0.4 \\
\hline Vientos Norte & 0.4 & 0.58 & 0.3 & 0.1 & 0 & 0.3 & 0.3 & 0.3 & 0.29 & 0.2 \\
\hline Vientos Sur & 0.8 & 0.4 & 0.8 & 0.36 & 0.4 & 0.36 & 0.5 & 0.6 & 0.53 & 0.7 \\
\hline OCE & 0.7 & 0.8 & 0.8 & 0.32 & 0.1 & 0.77 & 0.8 & 0.6 & 0.2 & 0.3 \\
\hline OCI & 0.6 & 0.85 & 0.7 & 0.42 & 0.3 & 0.88 & 0.7 & 0.6 & 0.28 & 0.4 \\
\hline OFI & 0.3 & 0.36 & 0.4 & 0.31 & 0.3 & 0.39 & 0.4 & 0.4 & 0.3 & 0.4 \\
\hline OFE & 0.7 & 0.7 & 0.7 & 0.3 & 0.3 & 0.69 & 0.6 & 0.3 & 0.33 & 0.4 \\
\hline Heladas & 0.8 & 0.15 & 0.6 & 0.57 & 0.2 & 0.22 & 0.6 & 0.6 & 0.57 & 0.2 \\
\hline Chubascos & 0.6 & 0.71 & 0.7 & 0.7 & 0.8 & 0.76 & 0.8 & 0.8 & 0.79 & 0.8 \\
\hline Nieblas & 0.8 & 0.41 & 0.6 & 0.71 & 0.7 & 0.42 & 0.7 & 0.7 & 0.42 & 0.8 \\
\hline Humidex & 0.2 & 0.33 & 0.4 & 0.4 & 0.1 & 0.4 & 0.4 & 0.2 & 0.2 & 0.2 \\
\hline THI & 0.2 & 0.61 & 0.6 & 0.5 & 0.2 & 0.6 & 0.5 & 0.4 & 0.2 & 0.4 \\
\hline $\mathbf{P}$ & 0.4 & 0.9 & 0.8 & 0.9 & 0.7 & 0.9 & 0.8 & 0.7 & 0.7 & 0.7 \\
\hline $\mathrm{Te}$ & 0.5 & 0.1 & 0.3 & 0.2 & 0.6 & 0.3 & 0.4 & 0.3 & 0.2 & 0.3 \\
\hline Cap & 0.2 & 0.88 & 0.7 & 0.59 & 0.4 & 0.81 & 0.5 & 0.4 & 0.5 & 0.5 \\
\hline Ep & 0.1 & 0.95 & 0.9 & 0.66 & 0.4 & 0.81 & 0.7 & 0.7 & 0.48 & 0.6 \\
\hline Tp & 0.3 & 0.95 & 0.8 & 0.5 & 0.5 & 0.86 & 0.8 & 0.7 & 0.6 & 0.6 \\
\hline Pap & 0.2 & 0.8 & 0.7 & 0.5 & 0.4 & 0.79 & 0.7 & 0.7 & 0.59 & 0.6 \\
\hline Tap & 0.5 & 0.8 & 0.8 & 0.4 & 0.7 & 0.75 & 0.7 & 0.7 & 0.51 & 0.6 \\
\hline Eep & 0.3 & 0.86 & 0.8 & 0.4 & 0.5 & 0.75 & 0.6 & 0.4 & 0.36 & 0.4 \\
\hline ICU & 0.2 & 0.92 & 0.4 & 0.41 & 0.2 & 0.86 & 0.3 & 0.4 & 0.42 & 0.4 \\
\hline Ind. Clim. Urb. & 9.7 & 17.1 & 15 & 12.2 & 9.6 & 16.3 & 13.1 & 12 & 11.15 & 11 \\
\hline
\end{tabular}

TST. Temperatura de Superficie Terrestre, OCE. Ola de Calor Estival, OCI. Ola de Calor Invernal, OFI. Ola de Frío Invernal, OFE. Ola de Frío Estival, H. Heladas, N. Nieblas, C. Chubascos, Cap. Calidad ambiental percibida, Ep. Percepción sobre la edificación, Tp. Percepción sobre el tránsito, Pap. Percepción sobre los problemas ambientales, Tap. Temperatura del aire urbano percibida, Eep. Eventos extremos meteorológicos percibidos, ICU. Intensidad de la Isla de Calor. 
De esta manera se definió el Indicador de Estado Actual de Bahía Blanca de la siguiente manera:

$$
\begin{gathered}
\text { IClimU }_{B B}=D_{e}+E_{v}+I_{c u}+T_{s t}+T_{a}+H_{r}+E v_{e x}+C C_{e}+C C_{i}+C A_{p} \\
+T V+A_{e d}+P A_{p}+S_{t}+E v_{e x p}
\end{gathered}
$$

donde $\operatorname{Iclim}_{b b}$ es el Indicador climático urbano de Bahía Blanca,

$\mathrm{D}_{\mathrm{e}}$ es la densidad edilicia en los distintos coberturas de la ciudad,

$\mathrm{I}_{\mathrm{cu}}$ Son los efectos de la Isla de Calor Urbana tanto en temperatura como en la humedad relativa,

$\mathrm{T}_{\text {st }}$ son los efectos de la Temperatura de Superficie Terrestre sobre las coberturas del suelo,

$\mathrm{T}_{\mathrm{a}}$, Temperatura del aire,

$\mathrm{H}_{\mathrm{r}}$, Humedad Relativa,

$\mathrm{Ev}_{\mathrm{ex}}$, es el efecto sobre la temperatura y la humedad relativa de los distintos eventos extremos analizados,

$\mathrm{CC}_{\mathrm{e}}$, Confort climático estival considerando el Humidex, el THI y el P,

$\mathrm{CC}_{\mathrm{i}}$, Confort climático de invierno teniendo en cuenta el índice Te,

$\mathrm{CA}_{\mathrm{p}}$, Calidad ambiental percibida,

$\mathrm{TV}$, tránsito vehicular percibido,

$\mathrm{A}_{\mathrm{ed}}$, Aumento de las edificaciones percibido,

$\mathrm{PA}_{\mathrm{p}}$, Problemáticas ambientales percibidas,

$\mathrm{S}_{\mathrm{t}}$, Sensación térmica urbana,

$\mathrm{EV}_{\text {exp }}$, Cantidad de eventos extremos percibidos,

Los resultados para la ciudad de Bahía Blanca oscilaron entre 9 y 18. Con este rango se identificó los valores 8-10 como Región I, 11-13 Región II, 14-16 Región III y $>16$ Región $I V$. Los resultados de este indicador fueron incorporados y analizados con el Sistema de Información Geográfica ArcGIS 9.3. El documento cartográfico generado tuvo en cuenta las distintas regiones que sirvieron de base para generar una propuesta sostenible para mitigar los efectos climáticos adversos.

\section{Resultados y discusión}

\subsection{Actores determinantes (drivers)}

El primer factor determinante es la expansión urbana. La ciudad de Bahía Blanca se expandió un $110 \%$ en los últimos 30 años (Ferrelli et al., 2016a). El crecimiento de las ciudades genera un desarrollo industrial, económico y social que impacta en el territorio urbano. Las consecuencias son el acelerado crecimiento de la población, la expansión de la ciudad, migraciones rural-urbanas y el incremento de los sistemas de transporte. Estos factores hacen que las ciudades se transformen en espacios dinámicos en constante crecimiento (Rojas Quezada et al., 2009). La ciudad de Bahía 
Blanca es la más grande de la región del sudoeste de la provincia de Buenos Aires. Esta característica es percibida por los migrantes como una potencial fuente de empleo, educación de nivel universitario y económico que pueden generar hasta flujos extranjeros de capitales (Brockerhoff, 1999).

Otro de los factores limitantes son la reducción de los espacios verdes. Las ciudades actuales presentan, en general, una urbanización de tipo difusa y extendida, policéntrica y basada especialmente en el uso de automóviles para el transporte. A raíz de ello se han aumentado las superficies construidas y se ha impermeabilizado la superficie. Como consecuencia se ha extendido el espacio urbano sobre los terrenos que anteriormente eran agrícolas o representaban paisajes naturales o rurales (Romero et al., 2007).

Las transformaciones generadas producto del crecimiento urbano han modificado la estructura y organización interna. La expansión de los usos residenciales, de los servicios y las industrias se han hecho sobre los espacios verdes disponibles. Históricamente estos últimos fueron utilizados para la recreación y contención del crecimiento urbano, a pesar de ello, los procesos de urbanización recientes comprometieron la preservación de las áreas verdes, especialmente en aquellas ciudades que no cuentan con una planificación o un plan de ordenamiento territorial (García y Guerrero, 2006).

Los espacios verdes pueden ser agrupados en espacios abiertos, lugares recreativos y de esparcimiento como los parques y plazas y sitios de acceso restringido. Estos últimos corresponden a los barrios-parque en donde los lugares altamente vegetados de tipo residencial adquieren estatus social (García y Guerrero, 2006). La relevancia física de estos espacios es que tienen una gran biodiversidad. Además mejoran la calidad del aire, reducen las emisiones de dióxido de carbono, la contaminación sonora, mejoran los procesos de infiltración, reducen la carga radiante de los ciudadanos y generan zonas de confort climático. En síntesis, mejoran la calidad ambiental de la ciudad y previenen posibles problemas de anegamientos, exceso de calor, etc. (Quattrochi y Ridd, 1998; Goetz et al., 2000; Dimoudi y Nikolopoulou, 2003; Weng et al., 2004; Sepulveda Moreno, 2006; Sarricolea et al. 2006; OltraCarrió et al. 2010). Actualmente, las funciones ecológicas de los espacios verdes se ven afectadas por distintos usos del espacio: turismo, recreación, poda de árboles, etc.

\subsection{Presiones (pressures)}

El clima urbano genera distintas presiones sobre el ambiente. La ciudad de Bahía Blanca presenta una marcada estacionalidad relacionada con la latitud y la circulación atmosférica global que le dan las características de un clima templado (Ferrelli, 2016). Se han observado cambios en todas las variables meteorológicas, algunas relacionadas con el crecimiento de la ciudad, como la disminución del viento y otras relacionadas a variaciones naturales del clima, como la alternancia de ciclos húmedos y secos (Ferrelli et al., 2015a). 
La mayor modificación producida por las ciudades es la generación de un clima local, denominado clima urbano. El mismo es uno de los elementos más significativos de las diferencias socio-ambientales en el interior de todas las ciudades de América Latina. Los distintos barrios, cuya localización está relacionada con los ingresos económicos, presentan diversas situaciones de usos y coberturas del suelo, generando modificaciones más o menos favorables para la calidad ambiental (Romero et al., 2007).

La presencia y crecimiento de las Isla de Calor Urbana se relacionan con la absorción y retención de la radiación solar (Oke, 1995). Esto depende de la naturaleza del suelo, la pendiente, la densidad de las construcciones, los materiales de construcción y la presencia (o no) de vegetación.

Las condiciones de sitio y el crecimiento desigual de la ciudad generan diferentes densidades edilicias. Estas impactan no sólo en las condiciones atmosféricas locales sino que además generan pérdida de suelos de alto valor productivo, pérdida de la capacidad de infiltración del acuífero, pérdida de biodiversidad, incremento de las presiones sobre áreas de interés ecológico y paisajístico y saturación de las vías de comunicación (Capelli de Steffens et al., 2005; Luber y McGeehin, 2008) Todo ello impacta sobre la calidad de vida de la población.

\subsection{Estado ambiental (state)}

El crecimiento de la ciudad de Bahía Blanca generó que la velocidad del viento se modifique por la forma y orientación de los edificios. Estos originan vórtices y torbellinos en ciertos sectores de la ciudad (Capelli de Steffens et al., 2005). El movimiento continuo del aire en la ciudad es fundamental para el bienestar térmico y las condiciones bioclimáticas de la población (García, 2005). La morfología urbana produce barreras que modifican este parámetro. El centro de la ciudad presenta una velocidad del viento menor que su periferia debido a la gran cantidad de obstáculos y barreras que debe atravesar (Piccolo et al., 1994).

En Bahía Blanca, la temperatura del aire se incrementó $0,9^{\circ} \mathrm{C}$ y la humedad relativa fue $6 \%$ menor en 2012 en relación con 1986 (Ferrelli et al., 2016a). Estos cambios en los parámetros meteorológicos estarían relacionados con el aumento del tránsito y la sustitución de suelo natural por edificaciones que modifican el albedo urbano e impactan sobre el clima de la ciudad (Sepulveda Moreno, 2006). Además, las zonas verdes urbanas de Bahía Blanca mostraron temperaturas más bajas en relación con las áreas construidas. Esto se debe a que estos espacios reducen la emisión de onda larga producida por las superficies construidas sometiendo a los habitantes a reducir su carga radiante (Dimoudi y Nikolopoulou, 2003).

El confort climático estableció que los sectores urbanos de Bahía Blanca que tienen mayor densidad de construcciones son los que presentaron mayor número de días de desconfort. Si bien las situaciones de desconfort son subjetivas a las personas y dependen en muchos aspectos de sus características físicas, sus extremos podrían poner de manifiesto situaciones críticas para la población, sobre todo a aquel grupo que realiza actividades al aire libre (Bustos y Piccolo, 2011; Ferrelli, 2016). Las 
situaciones adversas de confort en las ciudades de clima templado ponen de manifiesto la necesidad de contar con abundante cantidad de espacios verdes. El arbolado urbano de tipo caduco es el ideal para mitigar los efectos adversos del desconfort ya que provee de sombras en verano y en invierno, al perder el follaje permite el ingreso de la radiación, además mejora la calidad del aire y el paisaje urbano. Por otro lado, el viento adquiere importancia al momento de disipar las condiciones de calor del cuerpo durante los eventos cálidos (García, 2005).

\subsection{Impactos (impacts)}

Las condiciones actuales de las ciudades generan un entorno caracterizado por la presencia de abundantes edificios en altura que impactan en la pérdida de biodiversidad y degradación del ambiente humano. La constante demanda de espacios urbanos ha empujado a las ciudades a expandirse sobre terrenos rurales, generando en muchos casos asentamientos de alta vulnerabilidad térmica.

En los últimos años se ha producido una modificación de los ecosistemas como resultado de las actividades humanas y de las formas de intervención del hombre sobre la naturaleza (Hoffmann, 1996). La deforestación a gran escala, el cambio climático y el crecimiento desordenado de la población pusieron en riesgo comunidades biológicas y los servicios ecosistémicos que ellos proporcionan (Almeida-Leñero et al., 2007). Estos últimos son todos aquellos beneficios que la población obtiene de los ecosistemas. La reducción de los servicios ecosistémicos en las ciudades se relaciona principalmente con la impermeabilización de los suelos (Cram et al., 2007) y la reducción de la vegetación (Reyes Avilés y Gutierrez Chaparro, 2010). Teniendo en cuenta los autores anteriormente citados, se pueden mencionar:

- Reducción de la infiltración del agua por la impermeabilización de los suelos,

- Pérdida de soporte y suministro de nutrientes del suelo a las plantas,

- Reducción de las capturas de carbono,

- Disminución de las barreras contra la contaminación sonora,

- Aumento de la contaminación del aire por la reducción del arbolado urbano,

- Pérdida de la capacidad de reciclaje de compuestos orgánicos del suelo,

- Pérdida de la estética urbana para el desarrollo del turismo urbano o recreación,

- Pérdida de la biodiversidad,

- Destrucción del paisaje y los sitios para la recreación,

- Reducción de las sombras del arbolado urbano por exceso de poda o por inexistencia del mismo,

- Escasez de producción de oxígeno, etc.

Por otro lado, la Isla de Calor Urbano genera la pérdida de calidad de vida en las ciudades, destacándose como fuente principal el desconfort térmico que en ciertas ocasiones puede comprometer la vida de la población. Además estos fenómenos pueden causar enfermedades respiratorias debido a las oscilaciones térmicas diarias 
que favorecen la concentración de contaminantes atmosféricos (Patz, et al., 2005; Romero et al., 2007). La ICU además contribuye a aumentar la presencia de gases de efecto invernadero. Estos efectos se ven agravados por la retención del calor de las zonas construidas. Estas liberan la radiación absorbida en la noche, creando una fuente artificial de calor (Capelli de Steffens et al., 2005). La degradación del ambiente urbano afecta los recursos naturales, perjudicando la calidad del aire al aumentar la cantidad de bióxido de carbono que es perjudicial para la salud de la población. Como consecuencia, la actividad turística puede verse afectada como producto de la degradación ambientales (presencia de basura, deterioro de las costas, etc.) que afectan en la decisión del turista de visitar un sitio u otro (Li, 2003).

En ciertas ocasiones, los eventos extremos como las sequías, las inundaciones, las olas de calor y frío o las tormentas pueden desabastecer a la población de los servicios públicos. Entre ellos es posible mencionar la disponibilidad de agua, el acceso a la red eléctrica, gas natural, cortes de las redes de comunicaciones, etc. Según los ciudadanos de Bahía Blanca, las problemáticas ambientales que más efectos tienen sobre la ciudad son la presencia de olas de calor, de frío, modificaciones en los vientos, aumento de las tormentas severas y de las precipitaciones (Ferrelli et al., 2016a).

\subsection{Estado climático de la ciudad de Bahía Blanca}

Considerando los elementos anteriormente descritos se aplicó el Indicador Climático Urbano para definir áreas con características climáticas comunes. El mismo estableció 4 regiones en la ciudad de Bahía Blanca. Las mismas se identificaron como Región I, Región II, Región III y Región IV (Figura 3).

Se observaron diferencias significativas entre cada una de las regiones en lo que respecta a las variables meteorológicas. Las principales diferencias se presentan en la tabla 3 .

Tabla 3. Valores medios de las variables meteorológicas en cada una de las regiones.

\begin{tabular}{|c|c|c|c|c|}
\hline Variables & Región I & Región II & Región III & Región IV \\
\hline IClimU & 9.65 & 11.8 & 14.25 & 16.65 \\
\hline Temperatura ( $\left.{ }^{\circ} \mathbf{C}\right)$ & 17 & 17.2 & 17.8 & 18.2 \\
\hline Humedad (\%) & 62 & 62 & 58 & 53 \\
\hline Desconfort estival (días) & 10 & 25 & 36 & 40 \\
\hline $\begin{array}{c}\text { Desconfort invernal } \\
\text { (días) }\end{array}$ & 25 & 8 & 5 & 2 \\
\hline $\begin{array}{c}\text { Intensidad de la ICU } \\
\left({ }^{\circ} \mathbf{C}\right)\end{array}$ & 1 & 2.3 & 2.5 & 7 \\
\hline
\end{tabular}

Fuente: Elaboración propia. 
Figura 3. Regiones identificadas con el Indicador de Estado Actual de la ciudad de Bahía Blanca.

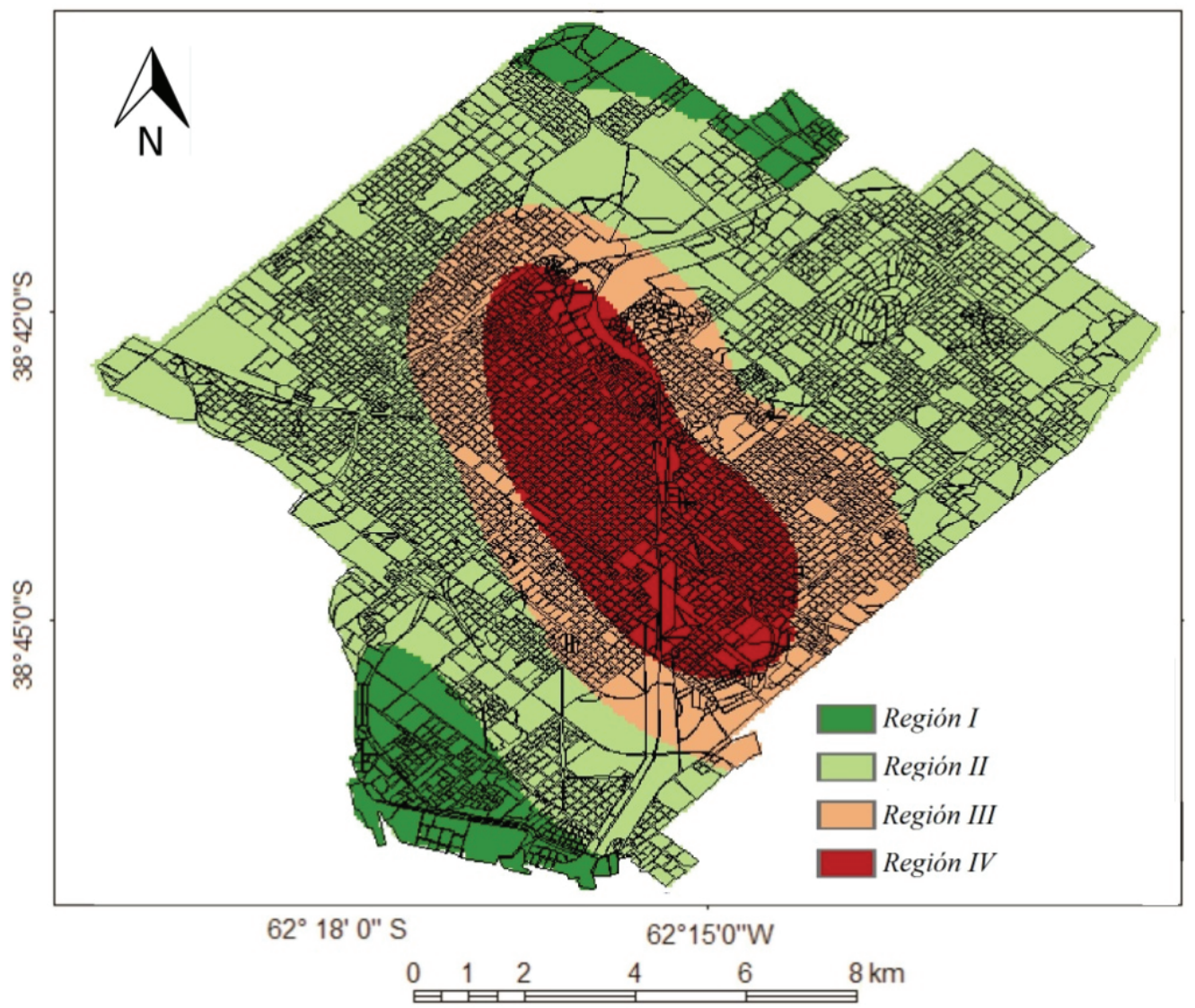

Fuente: Elaboración propia, 2016.

Región I. Corresponde a aquellas áreas localizadas en la periferia norte y noreste de la ciudad. Las mismas se caracterizan por presentar urbanizaciones poco densas, de escasa altura y densidad. En la zona costera, la humedad relativa tiene una función importante que es la de regular la temperatura del aire y generar situaciones de confort en casi todo el verano. En el norte y noreste, los efectos del viento norte tiene variaciones significativas en la humedad relativa, sin embargo, la ausencia o escasez de edificaciones no generan una fuente de calor artificial, por lo que su comportamiento es como el de la periferia urbana. Estas áreas presentan el menor 
número de días con desconfort en el verano. Sin embargo, son las áreas más desconfortables en invierno. El norte presenta un mayor valor de TST que el centro de la ciudad por su temprana exposición al sol, mientras que el sur tiene menores variaciones producto de la influencia del estuario. Los espacios verdes son abundantes.

Presenta grandes fluctuaciones de la temperatura y la humedad relativa durante los eventos extremos. Los vientos del norte aumentan la temperatura del aire y reducen significativamente su humedad, ocurriendo lo contrario durante los eventos de vientos del sur. Las olas de calor y de frío no afectan de forma intensa a estos espacios. La región I se caracteriza por la presencia de niebla y las heladas se registran sólo en la zona norte.

En cuanto a la percepción sobre estos espacios, el tránsito vehicular se consideró en un $54 \%$ que su aumento fue Medio y en un $44 \%$ que fue Alto. El $63 \%$ consideró que este espacio tiene un estado ambiental regular, mientras que el $37 \%$ lo caracterizó como Malo. Ello se debe en un $61 \%$ a la presencia de las industrias localizadas en el puerto de la ciudad y en menor medida (17\%) a la falta de conciencia social sobre el cuidado del ambiente. Además la zona norte ha aumentado en los últimos años la construcción de barrios residenciales incrementando consigo el tránsito vehicular. El $22 \%$ restante consideró que las actividades que ocasionan impactos sobre el ambiente son el aumento del tránsito vehicular, el crecimiento de las edificaciones y el loteo de espacios rurales para la urbanización. Los problemas ambientales percibidos demuestran que la escasez de arbolado urbano (55\%) es lo que más afecta a la población en el sector sur, seguido por cloacas y calles en mal estado (42\%) y presencia de basura en la vía pública (3\%). El $72 \%$ de la población percibió cambios en la temperatura, de ellos el $51 \%$ consideró un aumento moderado, un $39 \%$ un incremento alto y un $10 \%$ un crecimiento leve del parámetro. El mismo porcentaje de población percibió un incremento de los eventos extremos sobre estos espacios. De ellos el $50 \%$ percibió un aumento de las precipitaciones y el otro $50 \%$ un aumento de los eventos de olas de calor.

Región II. Son aquellas zonas urbanas de tipo residencial que rodean el centro de la ciudad junto con los barrios parque. La región tiene edificaciones bajas con arbolado urbano abundante en algunos sectores. La humedad relativa es mayor en el noreste y disminuye hacia el sudoeste. Los eventos extremos tienen efectos menores que en las Regiones III y IV. Los vientos del sur producen un aumento mayor de la humedad relativa en el sudoeste. Las heladas y nieblas se presentan sobre la noreste y oeste pero no observan sobre el sudoeste. La TST tiene variaciones significativas producto de la alternancia entre edificios y terrenos al descubierto. Los espacios verdes son numerosos en el noreste de la región.

En cuanto a la percepción de la población se encontró un caso particular al analizar esta región. En el sector noreste, coincidente con un barrio parque Patagonia, los encuestados manifestaron la necesidad de estar conformes con la cantidad de espacios verdes presentes $(51 \%)$. En el resto de la Región II, la población requirió un aumento de los mismos $(80 \%)$. El estado ambiental es Regular $(50,1 \%)$ y en menor medida Malo $(25,9 \%)$ y Muy Malo (16\%). Sólo el $8 \%$ considera que el estado 
ambiental de esta región es Bueno. E1 82,2 \% de los encuestados consideraron un aumento en el número de edificaciones, mientras que un $17,8 \%$ no lo hizo. En cuanto al tránsito vehicular, el $77 \%$ consideró que se observa un incremento del mismo de los cuales el $57 \%$ consideró un aumento Alto, un $29 \%$ Medio, $10 \%$ Muy Alto y $4 \%$ Bajo. En lo que respecta a los problemas ambientales, los ciudadanos las atribuyeron que la mayoría están relacionados a la falta de arbolado urbano y a la escasez de plazas $(51,6 \%)$, a las calles en mal estado $(25,8 \%)$, presencia de basura en la vía pública $(12 \%)$ y en menor medida a la contaminación del aire por mal olor $(10,6 \%)$. El 77,6 \% consideró un incremento en la temperatura de los cuales el $70 \%$ percibió un gran aumento del parámetro. Finalmente, el $89 \%$ notó un incremento de los eventos extremos, siendo mayoritario las olas de calor (48\%) y el aumento de las precipitaciones (38\%).

Región III. Se caracteriza por ser un espacio con presencia de edificios en altura pero más dispersos que en el centro. La densidad edilicia es mayor que en la Región II. En ambos espacios se encuentran los parques más importantes de la ciudad, Parque de Mayo y Parque independencia. La temperatura de superficie terrestre mantuvo un comportamiento uniforme sobre los espacios verdes pero fue irregular sobre las áreas con construcciones. Los espacios verdes son abundantes pero según los ciudadanos los mismos están en mal estado. Los eventos extremos manifestaron un impacto moderado sobre la región. No se genera la formación de nieblas ni heladas y los efectos de los vientos fueron iguales a los registrados en la Región IV.

Según la población, el estado ambiental de esta región es Regular $(65 \%)$ y en menor medida Malo (23\%) y Muy Malo (12\%). No calificaron en ninguno de los cuestionarios el estado ambiental como Bueno. El $77 \%$ consideró un aumento en las construcciones, de los cuales el $43 \%$ lo consideró Alto, el 30,5 \% Muy Alto y el 26,5 $\%$ Medio. El $92 \%$ consideró un aumento del tránsito siendo en su mayoría Alto (76 $\%)$ y en menor medida Medio (24\%). En cuanto a los problemas ambientales, los ciudadanos reconocieron en primer lugar a las calles en mal estado (32,3\%), seguido por mal estado de los espacios verdes $(26,5 \%)$, poda de árboles $(12,8 \%)$ y escasez de arbolado urbano (10,2 \%). El 18,2 \% restante fueron problemas como presencia de basura en las calles, fugas de gas, caños de agua rotos y contaminación del aire por mal olor. La población consideró un aumento de la temperatura (69\%), el mismo fue percibido principalmente como Alto (64\%), Moderado (20\%) y en menor medida Leve (16\%). El $72 \%$ de los encuestados notó un aumento de los eventos extremos, entre los que destacaron aumento de las precipitaciones (51\%), olas de calor (39\%), olas de frío $(8 \%)$ y aumento de las sequías $(2 \%)$.

Región IV. Corresponde a la zona central de la ciudad. Se presenta gran densidad de edificios en altura. La temperatura de superficie terrestre tiene los menores valores producto de la generación de sombras. Los espacios verdes son escasos, por lo que se dificulta la infiltración del agua y aumenta la escorrentía en épocas de lluvias.

Los eventos extremos de olas de calor generan un aumento en las temperaturas medias diarias. Las olas de frío reducen las temperaturas, pero sin embargo esta zona se presenta más cálida durante estos eventos. Los efectos de los vientos no son muy 
importantes en comparación con las otras regiones y no se presentan nieblas ni heladas. La Isla de Calor Urbana manifiesta su máximo valor en esta región siendo mayor a $7^{\circ} \mathrm{C}$. Es la más desconfortable durante el verano pero la que menos días con temperatura inferiores a $0{ }^{\circ} \mathrm{C}$ presenta en invierno.

Según la población el estado ambiental es Regular $(44 \%)$ y Malo $(36 \%)$ y en menor medida Muy Malo (11\%) y Bueno (9\%). El $82 \%$ consideró un aumento de las construcciones de edificios calificando a la misma como Alta (35\%), Muy Alta (32\%), Media (25\%) y Baja (8\%). El $91 \%$ consideró un aumento del tránsito siendo este Alto (66 \%) y Medio (34\%). Los problemas ambientales identificados fueron: escasez de arbolado urbano (27,3\%), calles en mal estado (25,8\%), escasez de desagües $(24,8 \%)$, contaminación sonora $(11,9 \%)$, contaminación del aire por mal estado de cloacas $(10,2 \%)$. En lo que respecta a la temperatura el $85 \%$ de la población percibió un incremento de la misma. Este fue Alto (54 \%), Moderado (39 $\%)$ y Leve $(7 \%)$. Finalmente el $91 \%$ consideró un aumento en el número de los eventos extremos. Los principales fueron incremento de las Precipitaciones (48\%), Olas de Calor (32\%), Aumento del viento (16\%) y Olas de frío (4\%).

\subsection{Propuesta sostenible para la ciudad (responses)}

Las propuestas fueron dirigidas y orientadas a cada una de las regiones generadas a partir de la aplicación del IClimU. Las mismas se resumen en la Tabla 4. Ellas están referidas al aumento y conservación de los espacios verdes; a la reducción de la contaminación sonora/acústica y del aire; a la reglamentación de las construcciones, re-pavimentación y control de desagües; y a la capacitación social.

Es importante realizar propuestas sostenibles dado que este concepto hace referencia a la importancia de asegurar las necesidades del presente sin comprometer a la capacidad de las futuras generaciones. Se incluyen dentro de este concepto a todas aquellas actividades que no perjudiquen o modifiquen un ecosistema. Los ciudadanos de la ciudad de Bahía Blanca consideraron que el estado actual de la ciudad es Regular. Esto se debe por un lado a los efectos negativos que causan las actividades industriales, al tránsito, la presencia de basurales a cielo abierto y el crecimiento de las construcciones. Por otro, se debe al aumento de la temperatura, a la ocurrencia de mayor número de tormentas, olas de calor, olas de frío, etc. Por tal motivo, fue necesario generar una propuesta sostenible que considerase las características climáticas y la percepción de la población en su conjunto. Para ello se tuvo en cuenta que las ciudades costeras presentan condiciones de tiempo muy variable por lo que necesitan de un plan de manejo sostenible (Reguero et al., 2015). 
Tabla 4. Propuestas sostenibles para mitigar los efectos adversos climáticos en Bahía Blanca.

\begin{tabular}{|c|c|}
\hline $\begin{array}{l}\text { Indicador } \\
\text { climático urbano }\end{array}$ & $\begin{array}{l}\text { Propuestas sostenibles para mitigar los efectos climáticos adversos en la ciudad de } \\
\text { Bahía Blanca }\end{array}$ \\
\hline Región I & $\begin{array}{l}\text { - } \text { Cortinas de viento } \\
\text { - Conservación de la vegetación nativa } \\
\text { - } \text { Restauración de vegetación nativa } \\
\text { - Conservación de la biodiversidad } \\
\text { - } \text { Planificación de las actividades de la población al aire libre durante el invierno. } \\
\text { - Capacitación ciudadana para la conservación del ambiente }\end{array}$ \\
\hline Región II & $\begin{array}{l}\text { - Mejorar la calidad de los espacios verdes } \\
\text { - Medidas de conservación de la biodiversidad } \\
\text { - Cortinas de viento } \\
\text { - Capacitación ciudadana para la conservación del ambiente }\end{array}$ \\
\hline Región III & $\begin{array}{l}\text { - } \\
\text { - } \text { Conservación de la biodiversidad } \\
\text { - } \text { Medidas de re-pavimentación y edificación } \\
\text { - Planificación de las actividades de la población al aire libre durante el verano } \\
\text { - } \\
\text { - Edificaciones de bajo impacto (calles y edificios). } \\
\text { - } \\
\text { - } \\
\text { - }\end{array}$ \\
\hline Región IV & 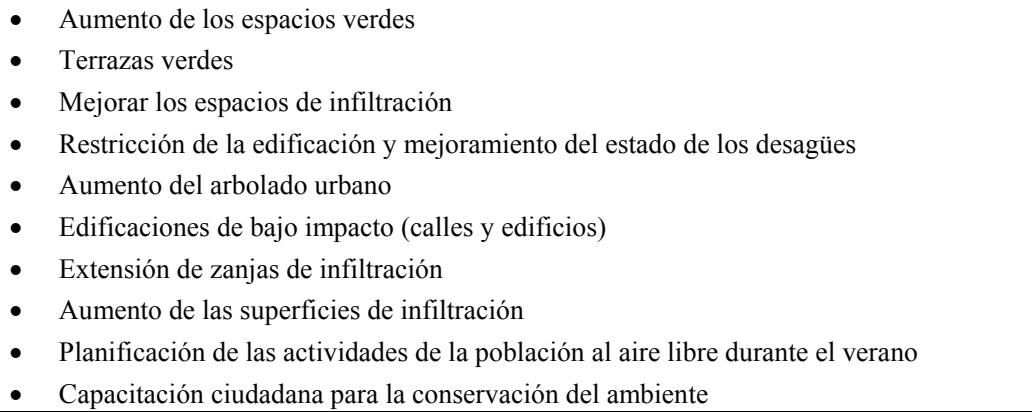 \\
\hline
\end{tabular}

Fuente: Elaboración propia.

Es importante realizar propuestas sostenibles dado que este concepto hace referencia a la importancia de asegurar las necesidades del presente sin comprometer a la capacidad de las futuras generaciones. Se incluyen dentro de este concepto a todas aquellas actividades que no perjudiquen o modifiquen un ecosistema. Los ciudadanos de la ciudad de Bahía Blanca consideraron que el estado actual de la ciudad es Regular. Esto se debe por un lado a los efectos negativos que causan las actividades industriales, al tránsito, la presencia de basurales a cielo abierto y el crecimiento de las construcciones. Por otro, se debe al aumento de la temperatura, a la ocurrencia de mayor número de tormentas, olas de calor, olas de frío, etc. Por tal motivo, fue necesario generar una propuesta sostenible que considerase las 
características climáticas y la percepción de la población en su conjunto. Para ello se tuvo en cuenta que las ciudades costeras presentan condiciones de tiempo muy variable por lo que necesitan de un plan de manejo sostenible (Reguero et al., 2015).

\subsubsection{Referidas a la conservación de los espacios verdes}

El aumento de los espacios verdes es necesario implementarlo en las Regiones III y $I V$ debido a que en ellas son escasos. En estas regiones la pavimentación es muy densa por lo que se mejoraría la capacidad de infiltración del agua. Además, la vegetación urbana tiene el rol de reducir la carga radiativa de los habitantes y las temperaturas extremas y mejorar la calidad del aire. En las Regiones III y IV, la densidad de edificaciones impide la circulación del aire debido a que los edificios modifican la circulación del viento, generando torbellinos y cambiando su dirección. Por este motivo es que en estos espacios se concentran los contaminantes y su depuración natural se dificulta.

Es importante para la Región $I V$ incorporar las terrazas verdes dado que representan una alternativa para incrementar el número de espacios verdes en las áreas altamente edificadas. Las mismas embellecen las infraestructuras desde el punto de vista estético y aumentan los espacios de infiltración, que en general son escasos en el centro de la ciudad de Bahía Blanca. Esta capacidad de infiltración permite filtrar el agua para reutilizarla con otros fines. Otra de las ventajas es que reduce el efecto invernadero que se genera en la atmósfera local como consecuencia de la baja depuración del aire, además reducen el uso de la energía fósil para la calefacción y aumentan el control del $\mathrm{CO}_{2}$ debido al aumento de la vegetación. Finalmente, colaboran con la disminución de la contaminación sonora ya que absorben el ruido de la ciudad (Jimenez et al., 2014).

Las Regiones I y II se ven muy afectadas por los eventos extremos de viento tanto del norte como del sur y por las olas de calor y de frío. Por ello, una alternativa para mitigar sus efectos son las cortinas de viento (Monelos y Peri, 1998, Peri, 1998). Las mismas están constituidas por vegetación que ayuda a reducir los efectos que los vientos tienen sobre los habitantes de la ciudad.

En toda la ciudad, se observó un número significativo de habitantes que percibieron a los espacios verdes como terrenos abandonados, con presencia de basura y generalmente en mal estado. Por ello, es que medidas de reacondicionamiento de los espacios verdes, dirigidas a toda la ciudad son necesarias para mejorar la calidad ambiental percibida por los habitantes. En este contexto, la importancia del cuidado y del aumento de los parques y plazas en las ciudades recae en la posibilidad de conservar y aumentar la biodiversidad. Los espacios verdes urbanos deben contar con vegetación nativa. Por ello es necesaria la implementación de un plan de reemplazo y cultivo de especies nativas. Esto aumenta la protección de la biodiversidad natural del espacio urbano. La participación de los ciudadanos en estos proyectos tanto en la conservación como en la vigilancia del cumplimiento es un requisito indispensable para la conservación de la biodiversidad (Sodhi et al., 2004; Duarte et al., 2008). 


\subsubsection{Referidas a la reglamentación de las construcciones}

En todas las regiones definidas con el IClimU, se observó un mal estado de las calles, por ello es necesario un proyecto de re-pavimentación que mejore la circulación de los habitantes. La ciudad de Bahía Blanca cuenta con ordenanzas que amparan este tema. Las ordenanzas número 18.012 (Utilidad Pública y Pago Obligatorio: Obra de Pavimentación varias calles de Bahía Blanca) y las 17.328 (Utilidad Pública y Pago Obligatorio: Pavimentación de varias calles de la ciudad de bahía Blanca, Etapa II, año 2013 (27 cuadras)) proponen un proyecto de repavimentación de distintos tramos de la ciudad. La primera de ellas fue decretada a partir de noviembre de 2014 y la segunda a partir de diciembre de 2013. Por otro lado, existe un Programa Municipal de Seguridad Vial (Bahía Conduce) vigente desde 2008 que incluye el desarrollo de obras de infraestructuras viales como la creación de puentes peatonales, peatonales, semi-peatonales y pavimentación de calles. La característica principal es la impermeabilidad de las calles y rutas en las ciudades. La infiltración y como consecuencia la recarga de los acuíferos se dificulta en estos espacios. La impermeabilización de las ciudades modifica además el flujo de la escorrentía. Por este motivo en las ciudades no sólo se infiltra menos agua sino que las características radiativas de los materiales con que se construyen las calles generan un aumento de la temperatura (Vázquez Rodriguez e Iturbe, 2014).

La escorrentía urbana genera la acumulación de contaminantes sólidos en distintos sitios de las ciudades que pueden afectar la salud de los habitantes, el crecimiento de las plantas y la calidad del suelo (Vázquez Rodriguez e Iturbe, 2014). El nuevo paradigma de las ciudades es el desarrollo de Edificaciones de Bajo Impacto. Se considera que estas edificaciones son importantes para las Regiones III y IV dado que el estado actual es de mucha impermeabilización y de alta densidad edilicia. En las futuras pavimentaciones, que puedan realizarse en las Regiones II y $I$ se debe tener en cuenta este tema. Estas edificaciones permiten la infiltración del agua. Se tratan de adoquines separados con espacios entre las juntas, celdas prefabricadas de concreto o de plástico que se rellenan con grava y los pavimentos porosos (Vázquez Rodriguez e Iturbe, 2014). Estos se enmarcan dentro de los denominados Sistemas de Drenaje Sostenible (Butler et al., 2008; Ávila, 2012).

El control y manejo de los desagües es un factor fundamental. Las precipitaciones son el evento extremo mayormente percibido por las personas en la ciudad por eso es necesario contar con un control de los desagües para evitar los procesos de anegamiento e inundación que podrían afectar a la población. En Bahía Blanca, el drenaje natural se modificó como consecuencia del parcelamiento de la ciudad generando una reconfiguración del escurrimiento natural. Las características del drenaje de la ciudad es sub-dendrítico. Las mejoras en la calidad del drenaje de la ciudad se relacionan con la extensión de zanjas de infiltración, aumento de las superficies de infiltración, aumento de los materiales de bajo impacto, construcción de terrazas verdes (en las Regiones III y IV) y reservorios (en las Regiones I y II) (Zapperi, 2014). 


\subsubsection{Referidas a la capacitación social}

El desarrollo local sostenible es el elemento que surge en el último tiempo para hacer referencia al crecimiento económico priorizando las cuestiones ambientales. Dentro de este contexto toman relevancia los actores del desarrollo local sostenible como lo son los gobiernos locales, las universidades, los ciudadanos, entre otros. Los actores sociales y los tomadores de decisiones son los encargados de que sea posible la implementación de una propuesta de desarrollo urbano sostenible dado que son quienes viven en el espacio urbano (Ahern, 2011; Ahern et al., 2014). Los actores sociales tienen participación activa en las políticas de sostenibilidad e implementación (Gray, 1989) así como también en su control y permanencia en el tiempo (Blomgren Bingham, 2006; Wolch et al., 2014; Reckien et al., 2014).

Dentro de este contexto se considera que para que la propuesta sostenible pueda llevarse a cabo es necesario considerar la participación activa de los actores sociales. Las autoridades disponen de ordenanzas y proyectos orientados a mejorar la calidad de vida, la circulación, la calidad de los espacios verdes, etc. Sin embargo, los ciudadanos no conocen estas normativas. Del cuestionario realizado, el 96,6\% manifestó desconocer medidas municipales para mejorar la calidad ambiental de los distintos sitios de la ciudad. El 3,4 \% identificó Reducción del uso de bolsas plásticas, separación de los residuos sólidos, Repavimentación de calles y sustitución de árboles enfermos por ejemplares jóvenes. Las medidas propuestas en este trabajo sólo podrán llevarse a cabo a partir de la educación de la población y concientización sobre los efectos adversos o impactos negativos que tiene el estado actual de la ciudad. Por ello, se considera necesaria una capacitación de la población para la conservación del ambiente urbano en toda la ciudad y debe incluir:

1. Generación de grupos de acción vecinal para controlar y concientizar a la población sobre la importancia de mejorar la calidad ambiental a partir del conocimiento del estado actual de la ciudad;

2. Concientizar a la población sobre los problemas ambientales que se generan a partir del exceso de basura en la vía pública;

3. Educar sobre los efectos adversos de los eventos extremos y cómo estos afectan a los distintos sectores de la ciudad;

4. Capacitar a la población sobre el término de desarrollo urbano sostenible, cómo llevarlo a cabo e incluir sus propuestas para mejorar la calidad ambiental de la ciudad;

5. Proponer a las autoridades locales el desarrollo de proyectos de terrazas verdes, el aumento de los espacios verdes, sistemas de drenaje sostenibles, separación y tratamiento de los residuos sólidos, tratamiento y control de los basurales para implementar una propuesta de manejo urbano en la ciudad de Bahía Blanca considerando las diferentes regiones identificadas con el IEA. 


\section{Conclusiones}

El diseño e implementación de un indicador climático urbano permitió delimitar 4 regiones en la ciudad de Bahía Blanca. El mismo estuvo compuesto por distintas variables meteorológicas. Además se basó en la percepción de la población de manera que el mismo incluyó datos meteorológicos registrados in situ y subjetivos derivados la percepción de la población.

La delimitación de la ciudad permitió orientar distintas medidas destinadas a mejorar la calidad de vida de la población y a mitigar los efectos adversos del clima. Por ello, se considera importante que en la puesta en marcha de la propuesta de manejo urbano sostenible participen activamente todos los actores sociales como los gobernantes y los ciudadanos. De igual manera, la concientización de la población sobre el estado actual de la ciudad de Bahía Blanca debería ser un tema prioritario. Con estas herramientas, la población local sabría cómo actuar antes diversos eventos extremos, cómo mejorar su calidad de vida, cómo cuidar el ambiente y sobretodo se podría generar un cambio en el comportamiento para que las medidas aplicadas perduren en el tiempo. Al tratarse de una herramienta sencilla, el método DPSIR y el Indicador Climático Urbano pueden aplicarse en otras ciudades del mundo para diseñar propuestas sostenibles para mitigar los efectos adversos que el clima puede tener sobre la calidad de vida de los espacios urbanos.

\section{Referencias bibliográficas}

Ahern, J. (2011): From fail-safe to safe-to-fail: Sustainability and resilience in the new urban world. Landscape and Urban Planning, 100 (4), 341-343.

Ahern, J., Cilliers, S. y Niemelä, J. (2014): The concept of ecosystem services in adaptive urban planning and design: A framework for supporting innovation. Landscape and Urban Planning, 125, 254-259.

Almeida-Leñero, L., Nava, M., Ramos, A., Espinosa, M., Ordoñez, M. J. y Jujnovsky, J. (2007): Servicios ecosistémicos en la cuenca del río Magdalena, Distrito Federal, México. Gaceta Ecológica número especial, 84-85, 53-64.

Ávila, H. (2012): Perspectiva del manejo del drenaje pluvial frente al cambio climático. Caso de estudio: ciudad de Barranquilla, Colombia. Revista de Ingeniería, 54-59.

Ballesteros Zapata, E. E. (2013): Clima y condiciones de confort en la ciudad de Villavicencio. Cap\&Cua, 10, 28-34.

Berenson, M. L. y Levine, D. M. (1996): Estadística Básica en administración: conceptos y aplicaciones. Prentice Hall Espanoamericana, S. A., segunda edición, México.

Blomgren Bingham, L. (2006): The new urban governance: processes for engaging citizens and stakeholders. Review of Policy Reasearch, 23 (4), 815-826.

Brockerhoff, M. (1999): Urban Growth in Developing Countries: A Review of Projections and Predictions Population and Development Review, 25, 757-778. 
Bustos, M. L. y Piccolo, M. C. (2011): Desconfort térmico en verano e invierno para la localidad de Pehuen Co, Argentina. Nimbus. Revista de meteorología, Climatología y Paisaje, 27/28: 63-76.

Butler, T.M., Lawrence, M.G., Gurjar, B.R., Van Aardenne, J., Schultz, M. y Lelieveld, J. (2008): The representation of emissions from megacities in global emission inventories. Atmospheric Environment, 42, 703-719.

Capelli de Steffens, A., Piccolo, M. C., Campo de Ferrera, A. (2005): Clima urbano de Bahía Blanca. Editorial Dunken, Buenos Aires, Argentina.

Cram S., Cotler, H., Morales L. M., Sommer, I. y Carmona, E. (2007): Identificación de los servicios ambientales potenciales en los suelos del paisaje urbano del Distrito Federal. Investigaciones Geográficas UNAM, 66, 81-104.

Cuadrat, J.M., Saz, M. A., Serrano, R. y Tejedor E. (2014): El clima del término municipal de Zaragoza en el contexto del cambio global. Departamento de Geografía y Orientación del Territorio, Universidad de Zaragoza, España.

CSD, Commission on Sustainable Development (2007): Indicators of Sustainable Development: Guidelines and Methodologies, Third Edition. New York, United Nations.

Dimoudi, A. y Nikopoulou, M. (2003): Vegetation in the urban environment: microclimatic analysis and benefits. Energy and Biuldings, 35, 69-76.

Duarte, C., Dennison, W., Orth, R. y Carruthers, T. (2008): The charisma of coastal ecosystems: addressing the imbalance. Estuaries and Coasts, 3, 233-238.

Ferrelli, F., Vitale, A. J. y Piccolo M. C. (2015a): Application of open source electronics for studying High Frequency Urban Heat Island. Anuario do Instituto de Geociencias- UFRJ, 38 (2), 70-80.

Ferrelli, F., Bustos, M. L., Huamantinco Cisneros, M. A. y Piccolo, M. C. (2015b): Utilización de imágenes satelitales para el estudio de la distribución térmica en distintas coberturas del suelo de la ciudad de Bahía Blanca (Argentina). Revista de Teledetección, 44, 31-42.

Ferrelli, F., Bustos, M.L., Piccolo, M.C. (2016a): La expansión urbana y sus impactos sobre el clima y la sociedad de la ciudad de Bahía Blanca, Argentina. Estudios Geográficos, En prensa.

Ferrelli, F., Bustos, M. L. y Piccolo, M. C. (2016b). Modificaciones en la distribución espacial de la temperatura y la humedad relativa como resultado del crecimiento urbano: el caso de la ciudad de Bahía Blanca, Argentina. Revista de Climatología, 16: 51-61.

Ferrelli, (2016): Análisis del clima local y micro-local de la ciudad de Bahía Blanca. Tesis de doctor en Geografía. Departamento de Geografía y Turismo, Universidad Nacional del Sur, $263 \mathrm{pp}$.

Franco Silva, F. J. y Pérez Salazar, L. M. (2008): Producción de ciudad, cotidianidad y culturas populares: una revisión preliminar. Investigación y Desarrollo, 16, 58-81.

Gabrielsen, P. Bosch, P. (2003): Environmental Indicators: Typology and use in Reporting. European Environmental Agency, Dinamarca.

García, J. (2005): Viento y arquitectura: el viento como factor de diseño arquitectónico. Editorial Trillas, México.

García S. y Guerrero M. (2006): Indicadores de sustentabilidad ambiental en la gestión de espacios verdes. Parque urbano Monte Calvario, Tandil, Argentina. Revista de Geografía Norte Grande, 35, 45-57. 
Goetz, S. J., Prince, S. D. y Small, J. (2000): Advances in Satellite Remote Sensing of Environmental Variables for Epidemiological Applications. Advances in Parasitology, 47, 289-307.

Gray, B. (1989): Collaborating: Finding common ground for multiparty problems. San Francisco: Jossey-Bass.

Hoffmann, R. (1996): Problemas y perspectivas de la valoración de recursos y procesos naturales: análisis de costo beneficio en áreas rurales del "Tercer Mundo". Economía Informa, 253, 29-44.

IPCC. Panel Intergubernamental de Cambio Climático. 2007. Cambio climático 2007. página web: www.ipcc.ch.

Jago-on, K.A.B., Kaneko, S., Fujikura, R., Fujiwara, A., Imai, T., Matsumoto, T., Zhang, J., Tanikawa, H., Tanaka, K., LeeTaniguchi, B. y Taniguchi, M. (2009): Urbanization and subsurface environmental issues: an attempt at DPSIR model application in Asian cities. Science of the Total Environment, 407, 3089-3104.

Kristensen, P. (2004): The DPSIR Framework. Comprehensive/Detailed Assessment of the Vulnerability of Water Resources to Environmental Change in Africa using River Basin Approach. UNEP, Kenia.

Li, H. (2003): Management of coastal mega-cities a new challenge in the 21st century. Marine Policy, 27, 333-337.

Luber, G. y McGeehing, M. (2008): Climate change and Extreme heat events. American Journal of Preventive Medicine 35, 429-435.

Mollinedo, C. L. (2006): Movilidad urbana sostenible: un reto para las ciudades del siglo XXI. Economía, sociedad y territorio, 6(22), 1-35.

Oltra-Carrio, R., Sobrino, J. A., Gutiérrez-Angonese, J., Gioia, A, Paolini, L. y Malizia, A. (2010): Estudio del crecimiento urbano, de la estructura de la vegetación y de la temperatura de la superficie del Gran San Miguel de Tucumán, Argentina. Revista de Teledetección, 34, 69-76.

Oke, T. 1995. Boundary Layer Climates. Routledge, London \& New York, Second Edition. Estados Unidos.

Patz, J.A., Campbell-Lendrum, D., Holloway, T. y Foley, J.A. (2005): Impact of regional climate change on human health. Nature, 438, 310-317.

Piccolo, M. C., Capelli de Steffens, A. y Campo de Ferreras, A. (1994): Efecto del viento en complejos edilicios bahienses. Revista Geofisica, 41, 5-19.

Quattrochi, D. A. y Ridd, M. K. (1998): Analisys of vegetation within a semi-arid urban environment using high spatial resolution airborne thermal infrared remote sensing data. Atmospheric Environment, 32, 19-33.

Reckien, D., Flacke J., Dawson R. J., Heidrich, O., Olazabal, M., Foley, A., Hamann, J. J., Orru, H., Salvia, M., De Gregorio Hurtado, S., Geneletti, D. y Pietrapertosa, F. (2014): Climate change responses in Europe: what's the reality? Analysis of adaptation and mitigation plans from 200 urban areas in 11 countries. Climatic Change, 122, 331-340.

Reguero, B. G., Losada, I. J. y Méndez, F. J. (2015): Las ciudades portuarias ante un clima cambiante. Dossier, 20-25. 
Replogle, M. A. (1991): Sustainable transportation strategies for Third World development. Transportation Research Record, 1294, 1-8.

Reyes Avilés, I. y Guitierrez Chaparro, J. J. (2010): Los servicios ambientales de la arborización urbana: retos y aportes para la sustentabilidad de la ciudad de Toluca. Revista de estudios urbanos, regionales, territoriales, ambientales y sociales, 1, 96-102.

Rojas Quezada, C. A.; Muñíz Olivera, I.; y García López, M.A. (2009): Estructura urbana y policentrismo en el área metropolitana de Concepción. Revista Eure, 105, 47-70.

Romero, H., Molina, M., Moscoso, C. y Smith, P. (2007): Cambios de usos y coberturas de los suelos asociados a la urbanización de las metrópolis chilenas. Anales de la Sociedad Chilena de Ciencias Geográficas, 194-198.

Sarricolea, P. y Romero Aravena, H. (2006): Cambios de uso y coberturas en el suelo entre 1998 Y 2004 y sus efectos sobre la configuración de la isla de calor de urbana de superficie de Santiago. Repositorio Académico de la Universidad de Chile, 1-4.

Sekovski, I., Newton, A. y Dennison, W. C. (2012): Megacities in the coastal zone: Using a driver-pressure-state-impact-response framework to address complex environmental problems. Estuarine, Coastal and Shelf Science, 96, 48-56.

Sepulveda Moreno, O. (2006): Problemas ambientales de Santiago: Isla de calor y edificios de vidrios. Boletín de Geografía, 24, 45-55.

Siple, P. A. y Passel. C. F. (1945): Measurements of Dry Atmospheric Cooling in Subfreezing Temperatures. American Philosophical Society, 177-199.

Sodhi, N.S., Koh, L.P., Brook, B.W. y Ng, P.K.L. (2004): Southeast Asian biodiversity: an impending disaster. Trends in Ecology \& Evolution, 19, 654-660.

Thom, E. C. (1959): The Discomfort Index. Weatherwise, 12, 57-60.

Vázquez Rodriguez G. e Iturbe U. (2014): Ciudades Permeables. ¿Cómo ves?, 183, 63-69

Vilani M. y Sanchez, L. (2013): Análise de Fourier e Wavelets aplicada à temperatura do ar em diferentes tipologias de ocupação. Revista Brasileira de Engenharia Agrícola e Ambiental, 17 (12), 1340-1346.

Weng, Q., Lu, D. y Shubring, J. 2004. Estimation of land surface temperature - vegetation abundance relationship for urban heat island studies. Remote Sensing of Environment, 89, 467-483.

Wolch, J. R., Byrne, J. y Newell, J. P. (2014): Urban green space, public health, and environmental justice: The challenge of making cities 'just green enough'. Landscape and Urban Planning, 125, 234-244.

Zulaica, L. y Tomadoni, M. (2015): Indicadores de sostenibilidad ambiental en el periurbano de la ciudad de Mar del Plata, Argentina. Anales de Geografía, 2: 195-216.

\section{Agradecimientos}

Los autores desean agradecer al Consejo Nacional de Investigaciones Científicas y Técnicas (CONICET) y al Departamento de Geografía y Turismo de la Universidad Nacional del Sur (UNS) por el financiamiento total de la presente investigación. 